\title{
Estudo da divergência genética entre linhas de suínos utilizando técnicas de análise multivariada
}

[Genetic divergence study of swine lines by multivariate analysis techniques]

\author{
R.A. Torres Filho ${ }^{1,3}$, R.F. Euclydes ${ }^{2,3}$, R.A. Torres ${ }^{2,3^{*}}$, P.S. Lopes $^{2,3}$, F.C. Breda ${ }^{1}$ \\ ${ }^{1}$ Estudante de pós graduação - Universidade Federal de Viçosa \\ ${ }^{2}$ Departamento de Zootecnia - Universidade Federal de Viçosa \\ Av. P.H. Rolfs, s/n \\ 33600-000 - Viçosa, MG \\ ${ }^{3}$ Bolsista do CNPq
}

\begin{abstract}
RESUMO
A divergência genética entre duas linhas de suínos da raça Large White foi avaliada utilizando-se técnicas de análise multivariada. Foram incluídas no estudo três características de desempenho - conversão alimentar, espessura de toucinho corrigida para $100 \mathrm{~kg}$ e ganho de peso médio diário para machos - e cinco reprodutivas - idade da porca ao primeiro parto, número de leitões nascidos vivos, número de leitões aos 21 dias e pesos da leitegada ao nascimento e aos 21 dias. Os dados foram agrupados em três arquivos. O primeiro constituiu-se de informações das características de desempenho avaliadas nos machos, o segundo continha informações reprodutivas, considerando parições até a quarta ordem e o último foi formado por informações de desempenho e reprodutivas das matrizes referentes ao primeiro parto. A análise de variância indicou a existência de divergência genética entre as duas linhas. Os resultados dos testes de união-interseção de Roy evidenciaram superioridade das linhas para características distintas, indicando a possibilidade de explorar a complementaridade em cruzamentos envolvendo as duas linhas. Foi encontrada diferença significativa pelo teste $\mathrm{F}$ para os escores obtidos pela aplicação da função discriminante linear de Fisher obtida na análise de cada arquivo que mostra, também, a existência da divergência genética entre as linhas de suínos.
\end{abstract}

Palavras-chave: suíno, características reprodutivas, desempenho, função discriminante de Fisher, teste Roy

\begin{abstract}
The genetic divergence between two Large White swine lines was evaluated by multivariate analysis techniques. Three performance traits (feed conversion, back fat thickness adjusted for $100 \mathrm{~kg}$ and average daily weight gain for males) and five reproductive traits (age at first farrowing, total number of piglets born alive, total number of piglets at 21 days, litter birth weight and at 21 days) were evaluated in this study. Data were grouped in three files. The first file consisted of male performance traits, the second of reproductive traits and the last of female performance and reproductive traits concerning the first farrowing. Multivariate analysis results showed a significant genetic divergence between lines. The Roy union-intersection test showed superiority of lines for different traits, suggesting that complementarity could be explored in crosses of the lines. Significant difference for the scores of Fisher linear discriminate function, in the analysis of each file also showed genetic divergence between the swine lines.
\end{abstract}

Keywords: swine, reproductive traits, performance traits, Fisher discriminant function, Roy Test

Recebido para publicação em 8 de outubro de 2003

Recebido para publicação, após modificações, em 8 de julho de 2004

*Autor para correspondência (corresponding author)

E-mail: rtorres@ufv.br 


\section{INTRODUÇÃO}

O estudo da divergência genética entre progenitores tem se constituído em uma ferramenta valiosa nos programas de melhoramento genético, pois, a partir dela, são identificadas as combinações híbridas de maior efeito heterótico, de tal forma que, nas gerações segregrantes, há maior possibilidade de recuperação dos genótipos superiores (Cruz e Regazzi, 1997). Os objetivos dos estudos de divergência genética foram citados por Piassi (1994).

Considerando que as características de importância econômica são correlacionadas, com magnitudes e sentido variáveis, a utilização de técnicas de análise multivariada é a mais recomendada no estudo da divergência genética, pois, quando existe interdependência entre elas, as análises individuais não apresentam a versatilidade de aproveitar as correlações existentes.

As análises univariadas referem-se ao comportamento dos grupos genéticos para cada uma das variáveis isoladas e não levam em consideração o conjunto de significâncias (James e McClloch, 1990, citados por Freitas, 1996).

Técnicas de análise multivariada foram empregadas no estudo da divergência genética na área animal em diferentes espécies (Sakagaguti, 1994; Piassi et al., 1995; Viana et al., 2000; Carneiro, 2002; Fonseca et al., 2002; Pires et al., 2002b). Em ampla revisão sobre análise de cruzamentos em suínos, Sancevero (1988) não fez referência à utilização de análise multivariada nos estudos de divergência genética. Freitas (1996) também não encontrou referências sobre a utilização de técnicas de análise multivariada em suínos.

Freitas et al. (1998) estudaram o emprego de técnicas de análise multivariada no estudo de divergência genética em suínos. Os cruzamentos foram realizados em duas fases distintas, e as técnicas avaliadas foram análise de variáveis canônicas, análise de agrupamento adotando a distância generalizada de Mahalanobis e o método de otimização de Tocher.

As técnicas de análise de variância multivariada, função discriminante linear de Fisher e análise de variáveis canônicas foram utilizadas para avaliar a divergência genética entre as raças de suínos Duroc, Landrace e Large White quanto às características reprodutivas por Fonseca et al. (2000) e quanto às características de desempenho por Pires et al. (2002a).

Cruz e Regazzi (1997) destacaram que, embora vários métodos multivariados pudessem ser aplicados na predição da divergência genética, a escolha do método mais adequado deve ser determinada pelo pesquisador, pela facilidade da análise e pela forma de obtenção dos dados.

A análise de variância multivariada (MANOVA) fornece resultados embasados na análise conjunta de todas as variáveis utilizadas. Rao (1952), citado por Demétrio (1985), afirma que a análise de variância multivariada consiste em analisar as variâncias e as covariâncias de variáveis correlacionadas por meio da comparação de matrizes de estimativas de variâncias e covariâncias. Posteriormente, podem-se aplicar testes de comparações múltiplas como o da união-interseção de Roy, para avaliar o desempenho dentro de cada variável considerada. Segundo Morrison (1967), o princípio da união-interseção de Roy deve ser utilizado como método de comparações múltiplas por ser a continuação lógica do teste do maior autovalor. Regazzi (2002) ressaltou a importância da função discriminante linear de Fisher (FDF), também conhecida como primeira variável canônica, que fornece o maior valor possível para $\mathrm{o}$ teste $\mathrm{F}$, entre todas as combinações lineares das variáveis envolvidas (Harris, 1975, citado por Regazzi, 2002).

Este trabalho teve como objetivo avaliar a divergência genética entre duas linhas de suínos da raça Large White quanto às características de desempenho e reprodutivas, utilizando técnicas de análise multivariada.

\section{MATERIAL E MÉTODOS}

Os dados utilizados são provenientes de duas linhas de suínos da raça Large White (LA e LB) em desenvolvimento, mantidas pelo programa de melhoramento genético da empresa Sadia SA, localizada no Estado de Santa Catarina, relativos ao período de 1991 a 1996. 
As características de desempenho foram conversão alimentar (CA), espessura de toucinho corrigida para $100 \mathrm{~kg}$ em mm (ETC) e ganho de peso médio diário em gramas (GPD). As características reprodutivas foram idade da porca ao primeiro parto (IPP), número de leitões nascidos vivos (NLN) e aos 21 dias (NL21), peso da leitegada ao nascimento (PLN) e aos 21 dias (PL21).

Como o manejo e o controle de informações foram diferenciados para cada sexo e houve interesse em avaliar a divergência genética quanto às características de desempenho e reprodutivas separada e simultaneamente, os dados foram agrupados em três arquivos. $\mathrm{O}$ arquivo 1, contendo 3635 registros, constituiu-se de informações das características de desempenho avaliadas nos machos; o arquivo 2, contendo 1702 registros, com informações reprodutivas, considerou parições até a quarta ordem; o arquivo 3, contendo 409 registros, com informações de desempenho das matrizes e reprodutivas, considerou somente o primeiro parto.

As análises estatísticas foram feitas utilizando o pacote estatístico Statiscal Analisys System (User's..., 1990). Como efeitos fixos, foram utilizados os grupos contemporâneos, formados pela combinação da semana do ano (1-48 à à $8^{\mathrm{a}}$ semana; $2-9^{\underline{a}}$ à $21^{\underline{a}}$ semana; $3-22^{\underline{a}}$ à $34^{\underline{a}}$ semana; 4-35 à $47^{\mathrm{a}}$ semana) com o ano de nascimento do animal. Para os arquivos 1 e 3 , as análises foram realizadas segundo o modelo:

$\mathrm{y}_{\text {tilr }}=\mu_{\mathrm{t}}+\mathrm{L}_{\mathrm{it}}+\mathrm{G}_{1}+\mathrm{e}_{\mathrm{tilr}}$,

em que $\mathrm{y}_{\text {tilr }}=$ valor observado da característica $\mathrm{t}$, na repetição $r$, da linha i, no grupo contemporâneo $1 ; \mu_{t}=$ média da característica $t$; $\mathrm{L}_{\mathrm{it}}=$ efeito da linha i na característica $\mathrm{t} ; \mathrm{G}_{1}=$ efeito do grupo contemporâneo 1 ; $\mathrm{e}_{\text {tilr }}=$ efeito do erro aleatório associado à observação $\mathrm{y}_{\text {tilr }}$.

Para o arquivo 2, as análises foram realizadas segundo o modelo:

$\mathrm{y}_{\mathrm{tikr}}=\mu_{\mathrm{t}}+\mathrm{L}_{\mathrm{it}}+\mathrm{O}_{\mathrm{k}}+\mathrm{e}_{\mathrm{tikr}}$,

em que $y_{\text {tikr }}=$ valor observado da característica t, na repetição $r$, da linha i, na ordem de parto k; $\mu_{t}, \quad L_{i t}=$ como descritos para o modelo anterior; $\mathrm{O}_{\mathrm{k}}=$ efeito da ordem de parto $\mathrm{k}$; $\mathrm{e}_{\mathrm{tikr}}=$ efeito do erro aleatório associado à observação $\mathrm{y}_{\text {tikr }}$.

Admite-se, nas análises, que os erros $\underset{\sim \mathrm{ir}}{\mathrm{e}}=\left[\mathrm{i}_{\mathrm{i} 1}^{\mathrm{e}}, \cdots, \underset{\sim \mathrm{ir}}{\mathrm{e}}\right]$ têm distribuição multinormal com vetor de médias nulo e matriz de variâncias e covariâncias $\sum$ comum a todas as combinações i e r. Os $\underset{\sim i r}{e}$ correspondentes às diferentes unidades experimentais são independentemente distribuídos.

Diferente da análise univariada, na multivariada (MANOVA) obtêm-se matrizes de somas de quadrados e produtos de dimensões $\mathrm{K} \times \mathrm{K}$, em que $\mathrm{K}$ é o número de características avaliadas por análise (no arquivo 1 tem-se $3 \times 3$, no 2 tem-se $4 \times 4$ e no 3 tem-se $7 \times 7$ ), sendo $\mathbf{A}, \mathbf{H}$ e $\mathbf{R}$ as matrizes de somas de quadrados e produto totais, tratamentos (linhas) e residuais, respectivamente. A hipótese testada pela MANOVA é a de igualdade dos vetores de média, ou seja, $\mathrm{H}_{0}: \underset{\sim 1}{\mathrm{~L}}=\underset{\sim 2}{\mathrm{~L}}$. Para testar essa hipótese, foi utilizado o teste do maior autovalor de Roy.

Em seguida, realizou-se a comparação de contrastes entre médias pelo teste da uniãointerseção de Roy, com a finalidade de identificar as fontes de variação que causaram a diferença apontada pela MANOVA. Contrastes entre os grupos genéticos foram construídos para cada característica. Assim, considerando a variável $\mathrm{k}$, para testar um contraste entre as médias $\left(\mathrm{Y}=\mathrm{c}_{1} \mathrm{~m}_{11}+\mathrm{c}_{2} \mathrm{~m}_{21}\right.$, com $\left.\sum_{\mathrm{i}=1}^{2} \mathrm{c}_{\mathrm{i}}=0\right)$, calcula-se a seguinte diferença mínima significativa:

DMS $=\sqrt{\frac{\theta_{\alpha}}{1-\theta_{\alpha}} \cdot \frac{S R_{\mathrm{r}}}{\overline{\mathrm{r}}} \cdot \sum_{\mathrm{i}=1}^{2} \mathrm{c}_{\mathrm{i}}^{2}}$,

em que $\theta_{\alpha}=$ valor crítico de Roy, no nível $\alpha$ de probabilidade com parâmetros s, m' e n'; $\mathrm{SQR}_{\mathrm{r}}=$ soma de quadrados do resíduo para a variável k; $\overline{\mathrm{r}}=$ aproximação do número de repetições, utilizando-se a média harmônica.

A função discriminante linear de Fisher, aplicada aos dados de observações, reduz o espaço $p$ dimensional a um espaço unidimensional, conservando a informação de variabilidade contida nos dados. A partir desse ponto, realizou- 
se uma ANOVA para o teste de hipótese de igualdade das linhas (tratamentos): $\mathrm{H}_{0}: \mathrm{L}_{1}=\mathrm{L}_{2}$ (igualdade dos efeitos de tratamento).

\section{RESULTADOS E DISCUSSÃO}

As médias e as diferenças mínimas significativas (DMS) por arquivo para o teste de uniãointerseção de Roy, a 5\% de probabilidade, encontram-se nas Tab. 1, 2 e 3.

Nas informações de desempenho dos machos (arquivo 1), observou-se diferença $(\mathrm{P}<0,01)$ entre os vetores de médias (linhas) pelo teste do maior autovalor de Roy. Pelo teste de união-interseção de Roy, foram observadas diferenças $(\mathrm{P}<0,01)$ entre as duas linhas, tendo a LA apresentado maiores valores para todas as características. Alguns dos interesses dos programas de melhoramento são voltados para maior ganho de peso e menor taxa de conversão alimentar. Assim, os resultados indicam a possibilidade de exploração da complementariedade entre as duas linhas para as características de desempenho.

Pires et al. (2002a), ao avaliarem as diferenças de desempenho entre suínos das raças Duroc, Landrace e Large White, encontraram diferenças entre as características espessura de toucinho e ganho de peso médio diário, contudo a conversão alimentar não foi avaliada. Os autores destacaram que a divergência genética entre as raças sugere que bons resultados de heterose e complementariedade podem ser obtidos com a produção de progênies oriundas de esquema de acasalamento tricross.

A função discriminante linear de Fisher (FDF) obtida foi: $\mathrm{FDF}=0,2914 \times \mathrm{ETC}+0,8620 \times \mathrm{CA}$ $+0,0890 \times$ GPDM. Houve diferença $(\mathrm{P}<0,05)$ entre as médias dos escores de LA e LB, que

foram de 104,66 e 93,85, respectivamente, constituindo, assim, mais uma evidência da divergência genética entre as duas linhas quanto às características avaliadas. Pires et al. (2002a), ao considerarem informações das raças Duroc, Landrace e Large White, referentes aos pesos dos leitões ao nascimento, aos 21 dias de idade e aos 70 dias de idade, ganho de peso médio diário, idade para atingir $100 \mathrm{~kg}$ e espessura de toucinho, encontraram diferenças significativas pelo teste $\mathrm{F}$ aplicado à FDF obtida. A raça Large White apresentou a maior média, e a Duroc a menor.

Tabela 1. Médias e diferença mínima significativa (DMS) de características de desempenho de machos de acordo com a linha da raça Large-White (LA e LB)

\begin{tabular}{lccc}
\hline Linha & ETC & CA & GPDM \\
\hline LA & $145,28 \mathrm{a}$ & $2,52 \mathrm{a}$ & $675,56 \mathrm{a}$ \\
LB & $112,74 \mathrm{~b}$ & $2,09 \mathrm{~b}$ & $664,83 \mathrm{~b}$ \\
Diferença & 32,54 & 0,43 & 10,73 \\
DMS & 1,96 & 0,02 & 5,48 \\
\hline
\end{tabular}

ETC $=$ espessura de toucinho $(\mathrm{mm})$ corrigida para $100 \mathrm{~kg} ; \mathrm{CA}$ = conversão alimentar; GPDM $=$ ganho de peso $(\mathrm{g})$ médio diário para machos.

Médias seguidas por letras distintas na coluna diferem entre si pelo teste Roy $(\mathrm{P}<0,05)$.

Tabela 2. Média e diferença mínima significativa (DMS) de características reprodutivas de acordo com a linha da raça Large-White (LA e LB)

\begin{tabular}{lcccc}
\hline Linha & NLN & NL21 & PLN & PL21 \\
\hline LA & $9,67 \mathrm{a}$ & $9,02 \mathrm{a}$ & $13,98 \mathrm{a}$ & $56,06 \mathrm{a}$ \\
LB & $11,03 \mathrm{~b}$ & $9,96 \mathrm{~b}$ & $14,77 \mathrm{~b}$ & $54,79 \mathrm{a}$ \\
Diferença & 1,36 & 0,94 & 0,79 & 1,27 \\
DMS & 0,42 & 0,40 & 0,55 & 2,41 \\
\hline NLN = número de leitões nascidos vivos; NL21 = número de \\
leitões aos 21 dias (desmame); PLN = peso (kg) da leitegada \\
ao nascimento; PL21 = peso (kg) da leitegada aos 21 dias. \\
Médias seguidas por letras, distintas na coluna diferem entre \\
si pelo teste Roy (P<0,05).
\end{tabular}

Tabela 3. Média e diferença mínima significativa de características de desempenho e reprodutivas de acordo com a linha da raça Large-White (LA e LB)

\begin{tabular}{lccccccc}
\hline & ETC & GPDF & IPP & NLN & NL21 & PLN & PL21D \\
\hline LA & $154,31 \mathrm{a}$ & 582,82 & $312,26 \mathrm{a}$ & $9,29 \mathrm{a}$ & $8,79 \mathrm{a}$ & 13,42 & $54,48 \mathrm{a}$ \\
LB & $112,44 \mathrm{~b}$ & 588,17 & $328,57 \mathrm{~b}$ & $10,69 \mathrm{~b}$ & $9,41 \mathrm{~b}$ & 13,09 & $48,51 \mathrm{~b}$ \\
Diferença & 41,87 & 5,35 & 16,31 & 1,40 & 0,62 & 0,33 & 5,97 \\
DMS & 4,38 & 7,53 & 4,84 & 0,69 & 0,61 & 0,85 & 3,83 \\
\hline
\end{tabular}

ETC = espessura de toucinho $(\mathrm{mm})$ corrigida para $100 \mathrm{~kg}(\mathrm{~mm})$; GPDF = ganho de peso $(\mathrm{g})$ médio diário para fêmeas; IPP = idade da porca (dias) ao primeiro parto; NLN = número de leitões nascidos vivos; NL21 = número de leitões aos 21 dias (desmame); PLN = peso da leitegada $(\mathrm{kg})$ ao nascimento; PL21 = peso da leitegada $(\mathrm{kg})$ aos 21 dias $(\mathrm{kg})$.

Médias seguidas por letras distintas na coluna diferem entre si pelo teste Roy $(\mathrm{P}<0,05)$. 
Considerando-se parições de até quarta ordem (arquivo 2), foram observadas diferenças $(\mathrm{P}<0,01)$ entre os vetores de médias (linhas) das características pelo teste do maior autovalor de Roy. Para as características número de leitões nascidos vivos e aos 21 dias de idade e peso da leitegada ao nascimento, houve diferença entre as linhas, com médias mais elevadas na LB (Tab. 2). Com relação ao peso da leitegada aos 21 dias, as duas linhas apresentaram comportamento semelhante. Fonseca et al. (2000) não encontraram diferenças para tamanho de leitegada ao nascimento, entretanto, para peso da leitegada ao nascimento, a raça Landrace apresentou maior média que a Large White. A raça Duroc comportou-se de maneira intermediária. Para as características tamanho da leitegada ao desmame e peso da leitegada aos 21 dias de idade, as raças Landrace e Large White não diferiram entre si e apresentaram médias maiores que a raça Duroc.

A FDF obtida foi: FDF $=-0,4616 \times$ NLNV $1,4933 \times \mathrm{NL} 21-0,2883 \times \mathrm{PLN}-1,9995 \times \mathrm{PL} 21$

A média dos escores para LA foi de 90,13 e para LB, de 85,32 $(\mathrm{P}<0,05)$. Fonseca et al. (2000) também encontraram diferenças para os escores obtidos pela aplicação da FDF. As raças Landrace e Large White apresentaram as maiores médias e não diferiram entre si; a raça Duroc foi a de menor média.

Os resultados obtidos pela MANOVA com as informações de desempenho e reprodutivas referentes ao primeiro parto das matrizes (arquivo 3) indicaram diferença $(\mathrm{P}<0,05)$ entre os vetores de médias da linhas. Semelhante às análises dos arquivos 1 e 2, foi observada divergência genética entre as duas linhas tanto para características de desempenho como reprodutivas. Os resultados encontrados indicam a possibilidade da exploração da heterose em cruzamentos entre o material genético avaliado, semelhante ao encontrado por Fonseca et al. (2000) e Pires et al. (2002a).

Para as características ganho de peso médio diário e peso da leitegada ao nascimento, não houve diferenças entre linhas (Tab. 3). Quanto à idade da porca ao primeiro parto e peso da leitegada aos 21 dias, houve diferenças entre linhas, sendo obtidos melhores valores na LA. Para espessura de toucinho, número de leitões nascidos vivos e aos 21 dias, a LB apresentou-se superior a LA. A superioridade de uma linha para algumas características e da outra linha para outras características indica a possibilidade da exploração da complementariedade entre as duas linhas.

A diferença dos resultados obtidos para ganho de peso médio diário nas duas análises pode ser atribuída ao fato de que, no arquivo 1, as informações são referentes a todos os machos nascidos no período e, no arquivo 3 , as informações referem-se apenas às fêmeas selecionadas para reprodução, constituindo-se em um volume de dados menor e préselecionado. As diferenças dos resultados obtidos para peso da leitegada ao nascimento e ao 21 dias podem ser atribuídas ao fato de que, no arquivo 2, as informações se referiam a partos até de quarta ordem e, no arquivo 3 , apenas ao primeiro parto.

A FDF obtida foi: $\mathrm{FDF}=0,8707 \times \mathrm{ETC}-0,3487$ $\times$ GPDF $-0,3514 \times$ IPP $-0,3681 \times$ NLN $-0,5167 \times$ $\mathrm{NL} 21+0,2735 \times$ PESNAS $+0,7008 \times$ PES21D.

A média dos escores para LA foi de $-144,73$ e para $\mathrm{LB}$ de $-193,90(\mathrm{P}<0,05)$. Os resultados foram semelhantes aos obtidos nas análises dos arquivos 1 e 2, evidenciando novamente a divergência genética entre as linhas quanto às características de desempenho e reprodutivas.

\section{CONCLUSÕES}

As duas linhas da raça Large White avaliadas apresentam divergência genética tanto para características de desempenho como reprodutivas, sugerindo que bons resultados de heterose e de complementariedade podem ser obtidos na exploração de matrizes F1 obtidas do cruzamento entre elas. A divergência genética encontrada poderia indicar $\mathrm{o}$ estudo da viabilidade da formação de uma nova linha a partir do cruzamento entre as duas linhas com o intuito de obter alta produtividade e variabilidade genética, que poderia ser explorada pela seleção nas gerações futuras. 


\section{REFERÊNCIAS BIBLIOGRÁFICAS}

CARNEIRO, P.L.S.; FONSECA, R.; PIRES, A.V. et al. Estudo da divergência genética entre linhagens de matrizes de frangos de corte por meio de análise multivariada. Arq. Bras. Med. Vet. Zootec., v.54, p.75-83, 2002.

CRUZ; CD.; REGAZZI, A.J. Modelos biométricos aplicados ao melhoramento genético. 2.ed. Viçosa, MG: Universidade Federal de Viçosa, 1997. 390p.

DEMÉTIO, G.C.B. Análise multidimensional para dados de cana-de-açúcar. 1985. 144f. Tese (Doutorado em Estatística) - Escola Superior de Agricultura "Luís de Queiroz", Universidade de São Paulo, Piracicaba, SP.

FONSECA, R.; PIRES, A.V.; LOPES, P.S. et al. Estudo da divergência genética entre raças suínas utilizando técnicas de análise multivariada. Arq. Bras. Med. Vet. Zootec., v.52, p.403-09, 2000.

FONSECA, R.; TORRES FILHO, R.A.; TORRES, R.A. et al. Avaliação de frangos de corte utilizando técnicas de análise multivariada: I Características de carcaça. Arq. Bras. Med. Vet. Zootec., v.54, p.525-529, 2002.

FREITAS, R.T.F. Estudo da divergência genética de suínos em cruzamentos, utilizando técnicas de análise multivariada. 1996. 152f. Tese (Doutorado em Zootecnia) - Universidade Federal de Viçosa, 1996. Viçosa

FREITAS, R.T.F.; SILVA, M.A.; LOPES, P.S. et al. Análise dialélica de características de leitegada de suínos usando-se variáveis canônicas. Rev. Bras. Zootec., v.27, p.700-7006, 1998.

LOPES, P.S.; FREITAS, R.T.F., FERREIRA, A.S. Melhoramento de suínos. Universidade Federal de Viçosa. 39p. (Caderno Didático, 37).

MORRISON, D.F. Multivariate statistical methods. New York: McGraw-Hill, 1967. 388p.

NASCIMENTO, J.D. Programa genético AGROCERES PIC. In: SIMPÓSIO NACIONAL DE MELHORAMENTO ANIMAL, 3., 2000, Belo Horizonte: UFMG, 2000. p.121-123.
PIASSI, M. Avaliação de do desempenho de linhagens de postura mantidas na Universidade Federal de Viçosa, em competição com marcas comerciais. 1994. 86f. Dissertação (Mestrado em Genética e Melhoramento) - Universidade Federal de Viçosa, Viçosa, MG.

PIASSI, M.; SILVA, M.A.; REGAZZI, A.J. et al. Estudo da divergência genética entre oito grupos genéticos de aves de postura, por meio de técnicas de análise multivariada. Rev. Bras. Zootec., v.24, p.715-727, 1995.

PIRES, A.V.; CARNEIRO, P.L.S.; TORRES FILHO, R.A. et al. Estudo da divergência genética entre seis linhas de aves Legorne utilizando técnicas de análise multivariada. Arq. Bras. Med. Vet. Zootec., v.54, p.314-319, 2002b.

PIRES, A.V.; FONSECA, R.; COBUCI, J.A., et al. Estudo da divergência genética entre as raças suínas Duroc, Landrace e Large White, utilizando técnicas de análise multivariada. Arch. Lat. Prod. Anim., v.10, p.81-85, $2002 \mathrm{a}$.

RAO, C.R. Advanced statistical methods in biometric research. New York: John Wiley \& Sons, 1952. 390p.

REGAZZI, A.J. Análise multivariada, Viçosa: Universidade Federal de Viçosa, Departamento de Informática, 2002 (Notas de aula).

SAKAGUTI, E.S. Utilização de técnicas de análise multivariada na avaliação de cruzamentos dialélicos em coelhos. 1994. 170f. Dissertação (Mestrado em Genética e Melhoramento) Universidade Federal de Viçosa, Viçosa, MG.

SANCEVERO, A.B. Aspectos genéticos do desempenho produtivo e reprodutivo do suíno em cruzamentos dialélicos. 1988. 152f. Tese (Doutorado em Zootecnia) - Universidade Federal de Viçosa, Viçosa, MG.

USER'S guide: statistics. Version 6, 4.ed. Cary: SAS Institute, 1990. 1686p.

VIANA, C.F.A.; SILVA. M.A.; PIRES A.V. et al. Estudo da divergência genética entre quatro linhagens de matrizes de frango de corte utilizando técnicas de análise multivariada. Rev. Bras. Zootec., v.29, p.1074-1081, 2000. 